\title{
An Insight into the Paradox of Selenium
}

\section{Ziad Moussa*}

Department of Chemistry, College of Science, United Arab Emirates University, UAE

*Corresponding author: Ziad Moussa, Department of Chemistry, College of Science, United Arab Emirates University, P.O. Box 15551, Al Ain, United Arab Emirates, Fax:+971-3-7134928, Tel:+971-3-7135396; Email: zmoussa@uaeu.ac.ae

\section{Editorial \\ Volume 3 Issue 2}

Received Date: June 24, 2019

Published Date: July 01, 2019

DOI: $10.23880 / \mathrm{macij}-16000140$

\section{Editorial}

\section{Introduction}

Did you know that out of the 90 elements that exist in nature, 25 constitute the human body? The largest of those elements is iodine, followed by molybdenum. Selenium ranks third. There has been a historical misconception surrounding the toxicity of selenium and it has been long overdue to set the record straight. The formal discovery of selenium dates back to the year 1818 and is attributed to the Swedish chemist J. J. Berzelius [1]. Shortly thereafter, the synthesis of numerous organoselenium derivatives, including selenides, alkyl selenols, selenoxides, and diselenides, was described by several research groups. Many such compounds were unpleasant to handle due their unstable or highly malodorous nature. These properties discouraged intensive research in the area of organoselenium chemistry and hampered the development of the field, leading to relatively slow progress for many decades. In 1927 elemental selenium was discovered to mediate the dehydrogenation of cyclic and polycyclic hydrocarbons to aromatic compounds [2], and shortly afterwards selenium dioxide were patented for its synthetic utility as an oxidant. These discoveries, along with the subsequent recognition that selenium is an essential trace nutrient for animals, prompted more intensive scrutiny of many aspects of the element and its derivatives, including their biological, physical, analytical, and chemical properties. A review describing the early organic chemistry of selenium appeared in 1963 [3] and the first comprehensive book on the subject, edited by Klayman and Gunther, was published in 1973 [4].

\section{Biochemistry of Selenium}

Selenium has a long-standing reputation of being a toxic element, often blamed for poisoning animals in areas where the soil selenium content is high. As early as 1916, the element was detected in human bone and tooth tissue samples and this discovery attributed an essential importance to it.

\begin{tabular}{|c|c|}
\hline Selenoprotein & Biochemical Function \\
\hline Glutathione peroxidase (GPX) & $\begin{array}{c}\text { Catalytic reduction of hydrogen peroxide, lipid and phospholipid } \\
\text { hydroperoxides }\end{array}$ \\
\hline Iodothyronine deiodinases & Synthesis and regulation of thyroid hormone \\
\hline Thioredoxin reductases & $\begin{array}{c}\text { Regulation of gene expression and maintenance of intracellular redox state. } \\
\text { Also required for cell viability and proliferation }\end{array}$ \\
\hline Mitochondrial capsule selenoprotein GPx & $\begin{array}{l}\text { Required for the protection of sperm cells from oxidative damage and is } \\
\text { critical for stability/motility of mature sperm }\end{array}$ \\
\hline Sperm nuclei GPx & Required for male fertility and sperm maturation \\
\hline Methionine sulfoxide reductase & Believed to be involved in regulating lifespan \\
\hline Selenoprotein W & Involved in skeletal and cardiac muscle metabolism \\
\hline Selenoprotein $P$ & Protects endothelial cells from oxidative damage by peroxynitrite \\
\hline Selenoprotein $\mathbf{N}$ & Maintenance of muscles; absence causes congenital muscular dystrophy. \\
\hline Selenophosphate synthetase, SPS2 & $\begin{array}{c}\text { Required for the synthesis of selenophosphate, an intermediate in the } \\
\text { biosynthesis of selenocysteine. }\end{array}$ \\
\hline 15 kDa Selenoprotein & Believed to protect prostate cells against carcinoma \\
\hline
\end{tabular}

Table 1: Mammalian selenoproteins and their biochemical functions. 


\section{Medicinal \& Analytical Chemistry International Journal}

In 1957, Schwarz and Foltz [5] identified it as an essential component of a dietary factor that protected rats from severe necrotic degeneration of the liver induced by feeding them a vitamin E deficient diet. This discovery sparked interest in the biochemical properties of selenium and prompted a number of research groups to investigate selenium deficiency syndrome in animals. These studies led to the discovery of two seleniumcontaining bacterial enzymes, glycine reductase and formate dehydrogenase in 1973 [6,7]. Concurrently, Flohé and Rotruck independently established the biochemical role of selenium in mammals by demonstrating its essential involvement in the active site of the antioxidant enzyme, glutathione peroxidase (GPx) [8]. The number of mammalian selenoenzymes has grown substantially since the discovery of GPx and to date, researchers have identified 35 selenoproteins. Several of these enzymes exhibit catalytic redox activity and are responsible for destroying harmful metabolic byproducts such as peroxides and peroxynitrites inside living cells. Others have structural and transport functions, such as production of hormones, regulation of gene expression, cell division and proliferation, protection of blood vessel lining, male fertility and suppressing the aging process. A number of highly active GPx mimetics have been reported in the literature [8-14]. Table 1 shows a selection of selenoproteins that play a crucial role in the maintenance and regulation of many biochemical pathways in mammalian organisms.

\section{Biochemical Role of Glutathione Peroxidase Enzymes}

A common feature of multicellular organisms is that they rely on aerobic cellular respiration to derive ATP.
The final electron acceptor for aerobic respiration is molecular oxygen, which is reduced by electrons that flow down the electron transport chain of mitochondria from NADH and FADH2. Unfortunately, the complete reduction of molecular oxygen to water proceeds via a univalent pathway to generate sequentially superoxide anion radical $\left(\mathrm{O}_{2} \cdot\right)$ and hydrogen peroxide $\left(\mathrm{H}_{2} \mathrm{O}_{2}\right)$ (Figure 1) [15]. The latter, if not destroyed, generates the highly reactive hydroxyl free radical $\left(\mathrm{OH}^{\circ}\right)$, which is widely believed to be the main species causing oxidative damage. The oxygen free radicals, radical anions and hydroperoxides, collectively termed reactive oxygen species are too reactive to be tolerated within living organisms and are responsible for a host of pathological processes including inflammation, DNA damage, neuron death, ischemia-reperfusion syndrome, and aging. Further, these agents generate other highly reactive entities (e.g. peroxynitrite, hypochlorous acid) that can attack DNA, membrane lipids and other cell components, thereby disrupting cellular function and integrity. The primary defense mechanism that organisms have evolved to protect against the deleterious actions caused by these reactive oxygen species is provided by two families of enzymes that catalytically destroy the intermediates of oxygen reduction. The superoxide dismutase enzymes protect against the reactive superoxide radical by catalyzing its conversion to hydrogen peroxide and oxygen [16]. This, however, causes the accumulation of hydrogen peroxide which itself is toxic. Fortunately, this peroxide and other hydroperoxides can be destroyed by the selenium-containing glutathione peroxidase enzymes which catalytically reduce such species to the corresponding water or alcohol.

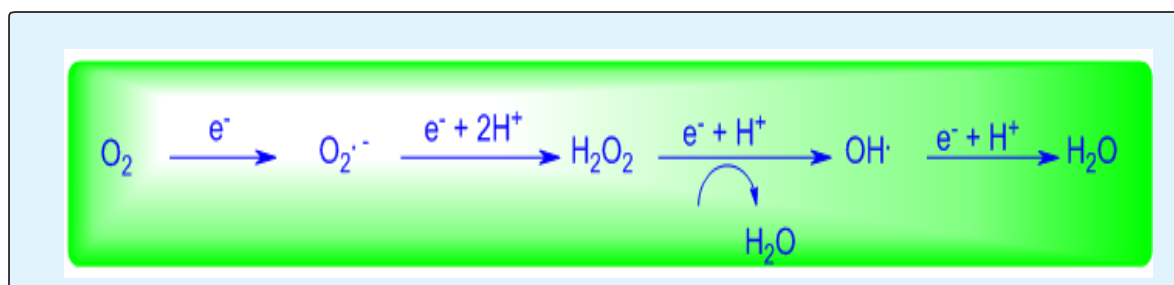

Figure 1: The univalent mechanism of oxygen reduction.

To date, four different types of selenium-containing glutathione peroxidase enzymes have been identified and classified as cytoplasmic GPx (cGPx), plasma GPx (pGPx), gastrointestinal GPx (giGPx), and phospholipid hydroperoxide GPx (PHGPx). Although they all catalyze the reduction of peroxides, the reactivity of these enzymes is highly dependent upon the peroxide and thiol cofactor which functions as the stoichiometric reducing agent in such processes. The most widely studied enzyme is the cytoplasmic GPx [17], which is present in nearly all tissues, albeit in different concentrations. The enzyme is a tetramer containing four identical subunits, each with a 
mass of approximately $21000 \mathrm{Da}$, and each containing one selenium atom. Selenium in the reduced enzyme exists as a selenocysteine residue and is stabilized through hydrogen bonding with proximal amido and imino groups of glutamine and tryptophan residues, respectively.

\section{Selenocysteine: The 21 ${ }^{\text {st }}$ Amino Acid}

The mechanism by which selenocysteine is incorporated into proteins in eukaryotic systems has not yet been fully elucidated and is less complete than in the case of the bacterial systems. Sunde and Evenson demonstrated that the carbon skeleton of the selenocysteine residue in eukaryotic GPx is derived directly from serine. Shortly afterwards, the open reading frame of the gene encoding GPx in eukaryotes was found to possess a TGA codon at a site which corresponds to the position of a selenocysteine residue in the subunit of the enzyme. Similarly, the gene encoding the prokaryotic selenocysteine-containing enzyme, formate dehydrogenase, was found to contain a TGA codon representing a selenocysteine unit in the gene product. The mechanism by which selenocysteine is biosynthesized is shown in Figure 2 [18]. First, the seryltRNA 1 is prepared from the amino acid serine and a unique tRNA whose anticodon is complementary to the TGA gene codon (UGA in the mRNA). The pyridoxal 5phosphate-containing enzyme 2 then undergoes transamination with seryl-tRNA 1 to afford complex 3 . Dehydration of the latter species and reaction of the resulting dehydroalanyl-tRNA product (4) with inorganic selenophosphate generates the corresponding pyridoxal 5-phosphate-containing selenoamino acid (5). This complex releases selenocysteyl-tRNA (6) and recycles the selenocysteine synthase enzyme (2). The former functions as a carrier molecule for selenocysteine and donates the group to the polypeptide chain in response to UGA codons during protein synthesis.

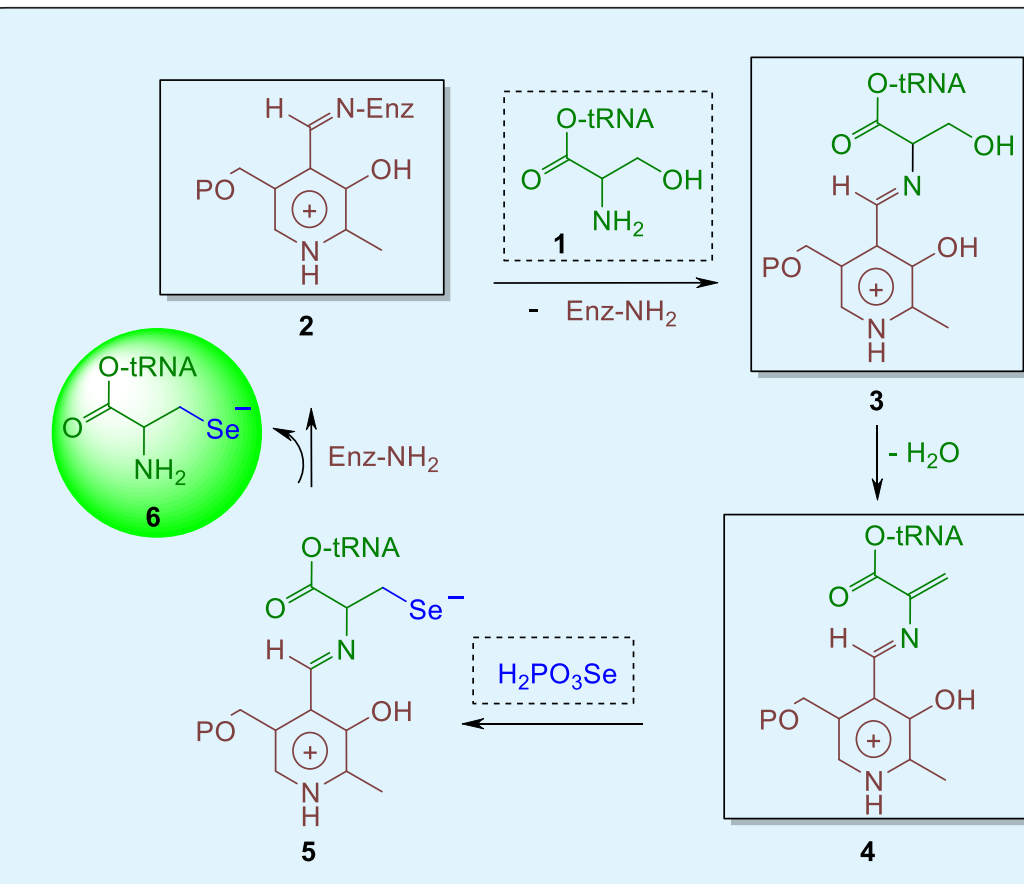

Figure 2: The biosynthesis of selenocysteine for eukaryotic GPx.

Figure 3 shows the mechanism by which cytoplasmic GPx catalyzes the reduction of peroxides at the expense of the cofactor, glutathione (GSH) [19]. First, the selenocysteine moiety (EnzSeH) in the enzyme is oxidized by peroxides to the corresponding selenenic acid (EnzSeOH), and the peroxide substrate is concomitantly reduced to the alcohol or water. In the second step, the thiol cofactor, glutathione (GSH), reacts with the selenenic acid species to produce a selenenyl sulfide intermediate (EnzSeSG) and water. Finally, the sulfur atom of the selenenyl sulfide gets attacked by a second molecule of glutathione, eliminating diglutathione disulfide (GSSG) and regenerating the selenol, thus completing the catalytic cycle. 


\section{Medicinal \& Analytical Chemistry International Journal}

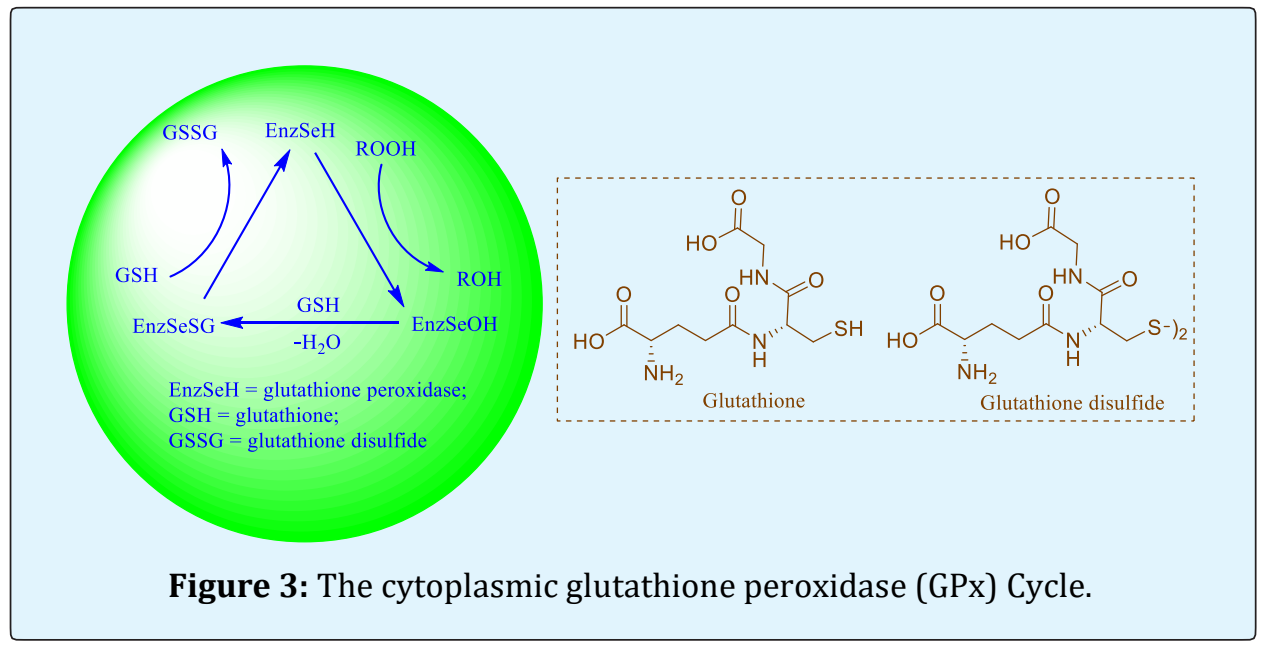

\section{So where is the Paradox ....}

While selenium deficiency is detrimental, high levels can result in toxicity. The paradox of selenium is reflected in the fact that it is essential, yet toxic. In fact, the element rose to stardom because of its toxic properties. Early in the twentieth century, livestock growers noticed that some of their animals manifested adverse symptoms after consuming loco weed which are plants that contain an unusually high amount of selenium. In humans, selenium toxicity occurs at doses higher than $400 \mu \mathrm{g} /$ day and is characterized by dermatitis, hair loss, diseased nails and peripheral neuropathy. The first indication of selenium toxicity is the development of a garlic breath and a dry skin. Then the fingernails start showing white patches, become brittle, and fall off!! Hair and nail loss occurs

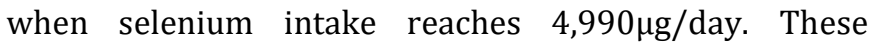

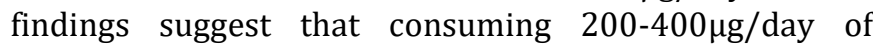
selenium as a pre-emptive strike against cancer is well tolerated, without side effects, in addition to one's dietary daily intake of selenium, which in the U.S. ranges from 60$110 \mu \mathrm{g} /$ day (in Europe it is $11-67 \mu \mathrm{g} /$ day). In 2000, the U.S. Food and Nutrition Board set the daily recommendation of selenium at $55 \mu \mathrm{g} /$ day for men and women, which is considered an adequate nutritional dose for 98 percent of the population. Such an amount supports the maximal expression of $\mathrm{GPx}$, which is regarded as fully discharging the nutritional effects of selenium. The U.S. Food and Nutrition Board set the tolerable upper intake level (UL) for selenium at $400 \mu \mathrm{g} / \mathrm{day}$. In China, authorities set the UL for no adverse effect level at $819 \mu \mathrm{g} /$ day and the low adverse effect level at $1540 \mu \mathrm{g} /$ day. China is known for having areas where the soil selenium level is severely deficient and other areas where the levels are toxic.

\section{Take your Selenium Supplement Today!}

There is a substantial amount of evidence that underlines the role of selenium in cancer prevention. Studies show that low selenium blood levels are associated with an increased risk of lung and prostate cancer. There is now very strong scientific evidence suggesting that selenium does indeed reduce the risk of cancer. Antioxidant activity and enhanced immune monitoring are two mechanisms that have been put forward to account for selenium's anticancer effect. Others include enhancement of programmed cancer cell death, regulation of cell proliferation, suppression of the growth of blood vessels supplying nutrients to the cancer cells, and inhibition of tumor cell spreading. The human heart does not function well without selenium. Inhabitants of the Keshan province of China, where soil selenium content is very low, develop a severe form of heart failure, a dilated cardiomyopathy known as Keshan's disease. Selenium supplements reverse it!! Selenium may help prevent coronary artery disease (atherosclerosis). Oxidation of low-density lipoprotein (LDL) is an initiating event in the inflammatory process that produces atherosclerotic coronary plaques and the antioxidant selenium-containing enzymes glutathione perioxidase and thioredoxin reductase can prevent the oxidation of LDL by using glutathione as a sacrificial reductant. Selenium boosts the immune system and has been shown to be effective in treating sepsis which involves blood stream infections. Research has shown that it increases the concentration of $\mathrm{T}$ cells throughout the body, both CD4 helper T cells and CD8 cytotoxic killer $\mathrm{T}$ cells. Even with an intake of a good dietary amount

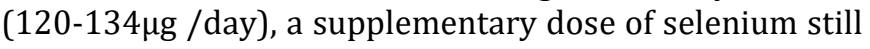
provides a considerable boost to the immune system. Selenium is also critical for male fertility and 


\section{Medicinal \& Analytical Chemistry International Journal}

reproduction. It is needed for the synthesis of testosterone and to maintain the structural integrity of sperm. As a final word, most of us do not get enough selenium in our diet. A supplementary selenium dose of $200 \mu \mathrm{g} /$ day, as selenomethionine or as sodium selenite / sodium selenate is physically well tolerated and has no side effects. It will contribute to an optimal healthy cancer-free lifestyle.

\section{References}

1. Berzelius JJ (1818) Ueber das selenium, Afhandl Fys Kemi Mineralogi 6: 42-144.

2. Diels 0, Karstens A (1927) Über dehydrierungen mit selen. Ber 60: 2323-2325.

3. Gosselck J (1963) Some aspects of the chemistry of organoselenium compounds. Angew Chem 2(11): 660-669.

4. Klayman DL (1973) Organic Selenium Compounds: Their Chemistry and Biology. Gunther WHH (Eds.), John Wiley and Sons, New York.

5. Schwarz K, Foltz C M (1957) Selenium as an integral part of factor 3 against dietary necrotic liver degeneration. J Am Chem Soc 79(12): 3292-3293.

6. Turner DC, Stadtman TC (1973) Purification of protein components of the clostridial glycine reductase system and characterization of protein $A$ as a selenoprotein. Arch Biochem Biophys 154(1): 366381.

7. Andreesen JR, Ljundahl LG (1973) Formate dehydrogenase of clostridium thermoaceticum: incorporation of selenium-75, and the effects of selenite, molybdate, and tungstate on the enzyme. Bacteriol 116(2): 867-873.

8. Flohé L, Günzler EA, Schock HH (1973) Glutathione peroxidase: a selenoenzyme. FEBS Lett 32(1): 132134.

9. Back TG \& Moussa Z (2005) Mimetics of the selenoenzyme glutathione peroxidase: novel structures and unusual catalytic mechanisms. Phosphorus Sulfur Silicon Relat Elem 180(3-4): $767-$ 776.
10. Back TG, Moussa Z (2005) Mimetics of the selenoenzyme glutathione peroxidase: novel structures and unusual catalytic mechanisms. Phosphorus Sulfur Silicon Relat Elem 180: 767-776.

11. Back TG, Moussa Z, Parvez M (2004) The exceptional glutathione peroxidase-like activity of di(3hydroxypropyl) selenide and the unexpected role of a novel spirodioxaselenanonane intermediate in the catalytic cycle. Angew Chem Int Ed Engl 43(10): 1268-1270.

12. Back TG, Moussa Z (2003) Diselenides and allyl selenides as glutathione peroxidase mimetics. remarkable activity of cyclic seleninates produced in situ by the oxidation of allyl $\omega$-hydroxyalkyl selenides. J Am Chem Soc 125(44): 13455-13460.

13. Back TG, Moussa Z (2002) Remarkable activity of a novel cyclic seleninate ester as a glutathione peroxidase mimetic and its facile in situ generation from allyl 3-hydroxypropyl selenide. J Am Chem Soc 124(41): 12104-12105.

14. Omar HS, El-Beshbishy HA, Moussa Z, Taha KF, Singab ANB (2011) Antioxidant activity of artocarpus heterophyllus lam. (jack fruit) leaf extracts: remarkable attenuations of hyperglycemia and hyperlipidemia in streptozotocin-diabetic rats. Scientific World Journal 11: 788-800.

15. Czapski G (1971) Radiation chemistry of oxygenated aqueous solutions. Annu Rev Phys Chem 22: 171-208.

16. McCord JM, Fridovich I (1998) Superoxide dismutase: the first twenty years (1968-1988). Free Radic Biol Med 5 (5-6): 363-369.

17. Flohé L (1985) The glutathione peroxidase reaction: molecular basis of the antioxidant function of selenium in mammals. Curr Top Cell Regul 27: 473478.

18. Böck A, Forchhammer K (1991) Selenocysteine synthase from Escherichia coli. Analysis of the reaction sequence. J Biol Chem 266(10): 6324-6328.

19. Ganther HE (1975) Selenoproteins. Chem Scripta 8: 79-90. 